CLINICAL REPORT

\author{
Y. Sunami \\ R. Koide \\ N. Arai \\ M. Yamada \\ T. Mizutani \\ K. Oyanagi
}

\section{Radiologic and Neuropathologic Findings in Patients in a Family with Dentatorubral- Pallidoluysian Atrophy}

\begin{abstract}
SUMMARY: We describe the cases of 2 patients, a father and his son, with DRPLA who underwent MR examinations prior to death and in whom postmortem examinations of the brain were obtained. MR imaging findings had the following features: 1) atrophy of the cerebellum and brain stem were the common findings, 2) high-signal-intensity lesions in the cerebral white matter and brain stem were observed on T2-weighted images in the patient with adult-onset DRPLA, 3) signal-intensity changes in the cerebral white matter were restricted to the periventricular white matter in the patient with juvenile-onset DRPLA, but these changes appear in the advanced stage, and 4) progressive cerebral atrophy was more marked in the patient with juvenile-onset DRPLA. In the patients with DRPLA, the abnormal high signal intensity of the cerebral white matter or brain stem on MR images reflect the loss of myelinated fibers. Cerebral atrophy mainly reflects atrophy of the neuropile.
\end{abstract}

ABBREVIATIONS: $A B C=$ avidin-biotin-peroxidase complex; DRPLA = dentatorubral-pallidoluysian atrophy; $K B=$ Klüver-Barrera
D RPLA is an autosomal dominant neurodegenerative disorder characterized by various combinations of myoclonus, epilepsy, cerebellar ataxia, choreoathetosis, dementia, and psychiatric symptoms. ${ }^{1}$ The clinical presentation varies depending on the age of onset. The cardinal features in adults are ataxia, choreoathetosis, and dementia, whereas cardinal features in children are ataxia, dementia, myoclonus, and epilepsy. In 1994, Koide et $\mathrm{al}^{2}$ reported that DRPLA is caused by $C A G$ repeat expansion in the DRPLA gene (atrophin 1) located on chromosome 12p13.31. The wide variation in clinical manifestations of DRPLA can be clearly explained on the basis of the degree of $C A G$ repeat expansion. ${ }^{3}$

Neuroradiologic findings of DRPLA are extremely specific. $^{4-8}$ Atrophic changes in the cerebellum and brain stem (in particular the pontine tegmentum) are the common MR imaging features of DRPLA. Diffuse high-signal-intensity lesions in the cerebral white matter are often observed on T2weighted images in patients with adult-onset DRPLA. In addition, high-signal-intensity lesions in the brain stem and thalamus can be observed on T2-weighted images. On the other hand, in patients with juvenile-onset DRPLA, marked progression of cerebral atrophy is often noted, whereas involvement of the cerebral white matter may appear in the advanced stage of the disease. To clarify the radiologic-pathologic correlation of DRPLA, we present the cases of 2 patients in a family who underwent MR imaging examinations before death and in whom postmortem examinations of the brain were performed.

Received April 12, 2010; accepted June 10.

From the Departments of Neurology (Y.S., R.K.) and Pathology (T.M.), Tokyo Metropolitan Neurological Hospital, Tokyo, Japan; Departments of Clinical Neuropathology (N.A.) and Neuropathology (K.O.), Tokyo Metropolitan Institute for Neuroscience, Tokyo, Japan; Department of Clinical Research (M.Y.), NHO Saigata National Hospital, Niigata, Japan; and Division of Neuropathology (K. O.), Department of Brain Disease Research, Shinshu University School of Medicine, Nagano, Japan.

Please address correspondence to Reiji Koide, MD, Department of Neurology, Tokyo Metropolitan Neurological Hospital, 2-6-1 Musashidai, Fuchu, Tokyo 183-0042, Japan; e-mail: reiji@tmnh.fuchu.tokyo.jp

DOI 10.3174/ajnr.A2252

\section{Case Reports}

\section{Patient 1}

His initial symptom of gait instability developed at 56 years of age. At 60 years of age, neurologic examination demonstrated cerebellar ataxia, orolingual dyskinesia, and choreic movement of the limbs. Cognitive changes and cerebellar ataxia gradually worsened, and he could not stand or walk without assistance at 62 years of age. At 63 years of age, his intellectual function was moderately disturbed (Mini-Mental State Examination score, 20/30), and personality changes and behavioral disinhibition were observed. The patient died at 65 years of age because of aspiration pneumonia.

\section{Patient 2}

This patient was a son of patient 1 . His symptoms began at of 19 years of age with grand mal seizures. On examination at 29 years of age, marked cerebellar ataxia and myoclonus of the limbs were observed. His intellectual function was severely disturbed (his intelligence quotient was 35 by the Suzuki-Binet test, which is a Japanese version of the Binet test), and personality changes and behavioral disinhibition were observed. Treatment-resistant seizures often occurred. At 31 years of age, he became bedridden and was unable to communicate. The patient died at 40 years of age because of aspiration pneumonia.

DNA analysis for DRPLA was performed in both patients by using a method reported previously. ${ }^{2}$ The number of expanded $C A G$ repeats was 65 and 70 in patients 1 and 2, respectively (normal range, from 7 to 34 repeats).

\section{MR Imaging}

MR imaging was performed by using a $0.5 \mathrm{~T}$ MR imaging scanner (Vista; Picker International, Cleveland, Ohio) and a 1.5T MR imaging scanner (Signa; GE Healthcare, Milwaukee, Wisconsin) in patients 1 and 2, respectively. MR images were obtained with spin-echo sequences (TR/TE $=600 / 26 \mathrm{~ms}$ for T1weighted images; TR/TE $=2000 / 100 \mathrm{~ms}$ for T2-weighted 

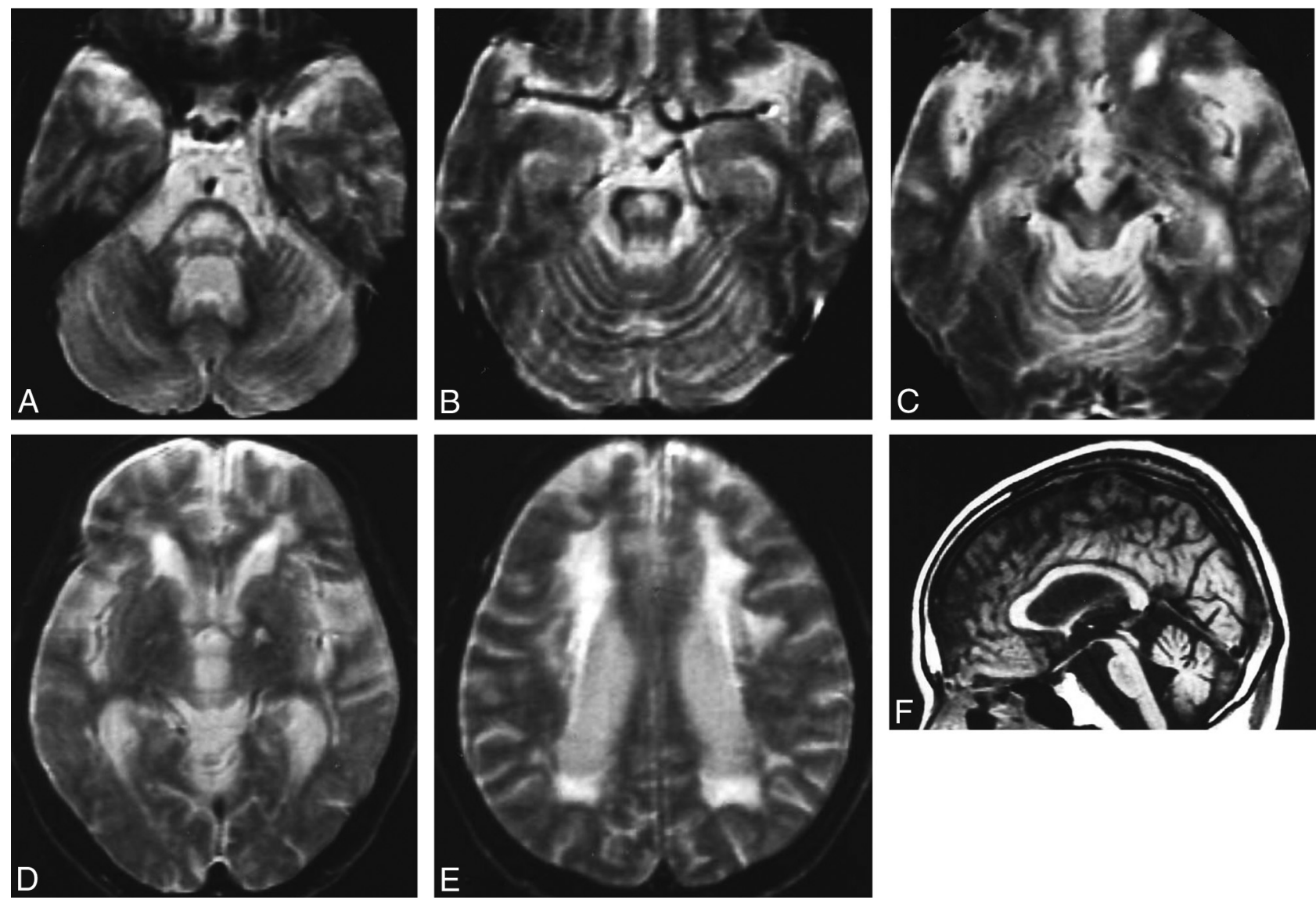

Fig 1. MR images of patient 1 (with adult-onset DRPLA). A-E, The T2-weighted axial images obtained at 60 years of age show high-signal-intensity lesions in the middle and upper pons, midbrain tegmentum, and cerebral white matter, in addition to a left pallidal high-signal-intensity spot due to an old lacunar infarction (D). $F, A$ T1-weighted midsagittal image shows atrophy of the brain stem and cerebellum

images in patient 1 , and $\mathrm{TR} / \mathrm{TE}=4000 / 100 \mathrm{~ms}$ for $\mathrm{T} 2$ weighted images in patient 2).

\section{Pathologic Examinations}

The brains were fixed in 10\% formalin after postmortem examination of each patient performed several hours after death. Tissue samples were taken from various parts of the central nervous system, and 2 age-matched controls showing no evident neurologic symptoms or significant neuropathologic findings in the central nervous system were also examined. Tissues were dehydrated in a graded ethanol series, embedded in paraffin, and cut into 6 - $\mu \mathrm{m}$-thick sections. The sections were stained with hematoxylin-eosin, $\mathrm{KB}$, Bodian, and Holzer's. Immunohistochemical analysis was performed by using $\mathrm{ABC}$ with a Vectastain $\mathrm{ABC}$ kit (Vector Laboratories, Burlingame, California). The primary antibodies used were an anti-phosphorylated neurofilament monoclonal antibody (SMI31; dilution, 1:1000; Covance, Princeton, New Jersey) and an anti-glial fibrillary acidic protein polyclonal antibody (GFAP; dilution, 1:500; DAKO, Glostrup, Denmark). Nonspecific binding of the biotin/avidin system reagents was blocked by pretreating the sections with a blocking solution from the kit (Vector Laboratories). The sections were subjected to peroxidase reaction by using freshly prepared $0.02 \% 3,3^{\prime}$-diaminobenzidine tetrahydrochloride and $0.005 \%$ hydrogen peroxide in $0.05-\mathrm{mol} / \mathrm{L}$ Tris- $\mathrm{HCl}$ buffer, $\mathrm{pH} 7.6$, for 10 minutes at room temperature. As antibody controls, primary antisera were either omitted or were replaced with normal rabbit or mouse serum. The stained sections were examined by light microscopy.

\section{MR Imaging Findings}

\section{Patient 1}

MR images obtained at 60 years of age showed atrophy of the cerebellum, midbrain, and pons associated with dilation of the fourth ventricle (Fig 1F). T2-weighted images showed high-signal-intensity lesions in the pons, midbrain tegmentum, and cerebral white matter (Fig $1 A-E)$.

\section{Patient 2}

T2-weighted axial MR images obtained at 35 years of age showed marked atrophy of the pons, cerebellum, and both hemispheres of the cerebrum (Fig $2 A-C$ ). Minimal high-signal-intensity lesions in the cerebral white matter were observed at this point in time (Fig 2C). On T2-weighted MR images at 39 years of age, marked progression of atrophic changes was demonstrated in the cerebellum, brain stem, and both hemispheres of the cerebrum (Fig 2D, $-F$ ). Additionally, high-signal-intensity lesions in the cerebral white matter were clearly observed (Fig $2 F)$. 

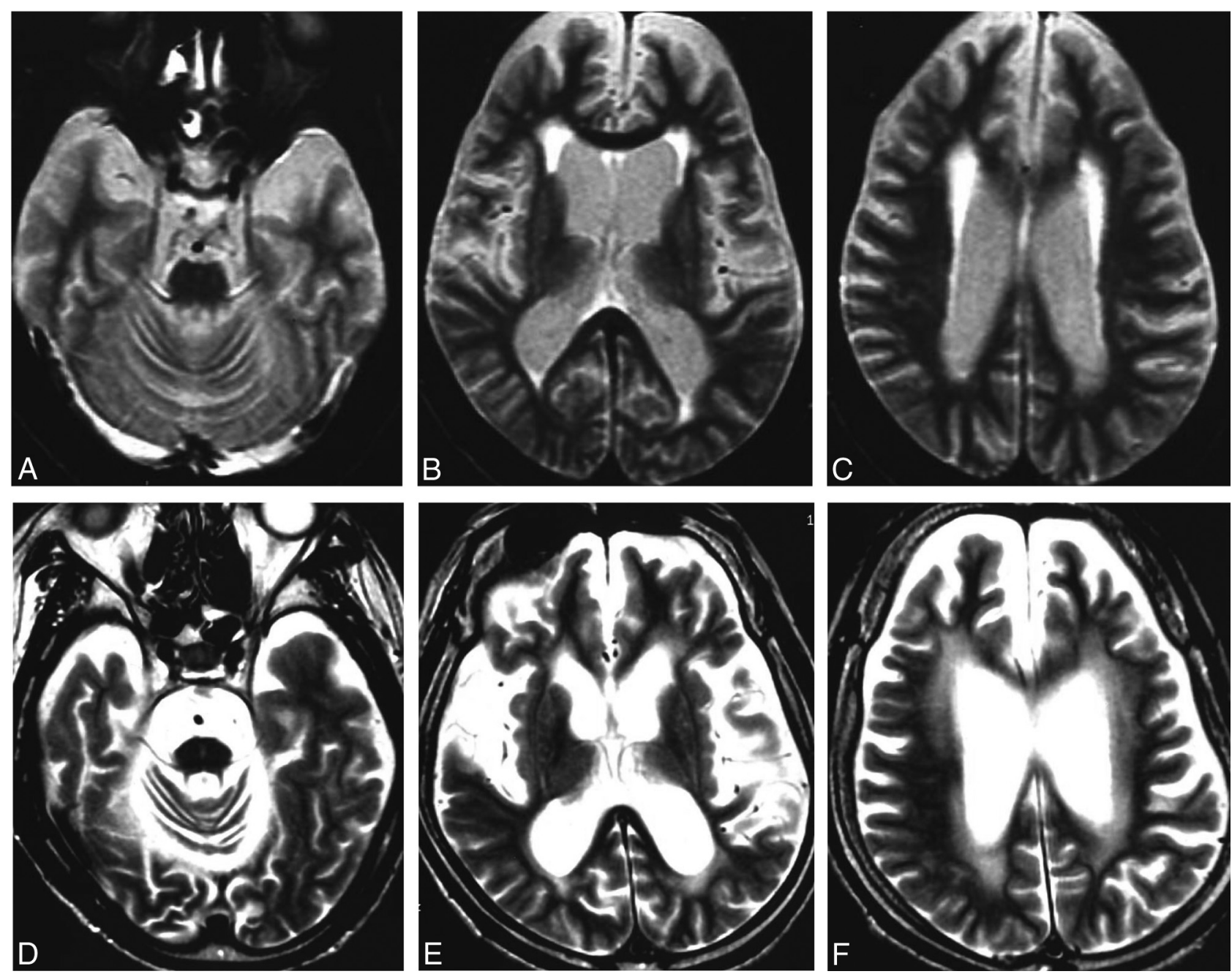

Fig 2. MR images of patient 2 (with juvenile-onset DRPLA). A-C, The T2-weighted axial images obtained at 35 years of age show marked atrophy of the pons, cerebellum, and cerebral hemispheres. High-signal-intensity lesions are observed only in the periventricular white matter at this point in time $(C)$. $D-F$, On T2-weighted MR images obtained at 39 years of age, marked progression of atrophic changes is demonstrated in the cerebellum, brain stem, and cerebral hemispheres. Additionally, high-signal-intensity lesions in the cerebral white matter are clearly observed $(F)$.

\section{Pathologic Findings}

\section{Patient 1}

The fresh brain weighed $1218 \mathrm{~g}$. There was marked atrophy of the cerebellum and brain stem and mild atrophy of the frontal lobe. Microscopic examination showed moderate neuronal loss in the dentate nuclei, subthalamus, and external segment of the globus pallidus. Holzer staining revealed fibrillary gliosis in the external segment of the globus pallidus. Cerebral cortical neurons showed slight shrinkage without evident loss (Fig 3F, -G). Marked accumulation of phosphorylated neurofilaments was observed in several cortical neurons, especially in the fifth layer (Fig $3 H$ ) compared with the control (Fig $3 A-C$ ). Diffuse myelin pallor of the cerebral white matter was observed (Fig 3F, $I$, and $J$ ) compared with the control (Fig $3 D,-E)$, but no atherosclerotic changes of the vessels in this region were found. In the pons, marked loss of myelinated fibers was found in the base (Fig $4 D,-F$ ) compared with the control (Fig $4 A-C$ ). Neurons in the pontine nuclei were slightly shrunken, but their attenuation was well-preserved. Infiltration of macrophages was not observed (Fig 4E). Marked loss of myelinated fibers was also found in the mid- brain, though neurons in the substantia nigra and red nucleus were well preserved.

\section{Patient 2}

The fresh brain weighed $1024 \mathrm{~g}$. There was marked atrophy of the cerebrum, predominantly in the frontal lobe and brain stem. Microscopic examination showed neuronal loss with astrocytosis in the dentate nuclei, subthalamus, and external segment of the globus pallidus. The width of the cerebral cortical ribbon appeared small, and the volume of the cerebral white matter was markedly reduced (Fig $3 K$ ). Cortical neurons were markedly shrunken, and the neuropile was markedly atrophic in every layer (Fig $3 L$ ). Several cortical neurons in the fifth layer showed accumulation of phosphorylated neurofilaments (Fig $3 M$ ). Loss of myelinated fibers was also observed in the cerebral white matter (Fig $3 K, N$, and $O$ ), but the loss was clearly slight in comparison with that in patient 1 . The pons was severely atrophic; the neurons in the pontine nucleus were shrunken, but their attenuation appeared to be increased due to atrophy of the neuropile (Fig $4 G,-H$ ). The attenuation of myelinated fibers appeared preserved in the pontine base (Fig 4I). 

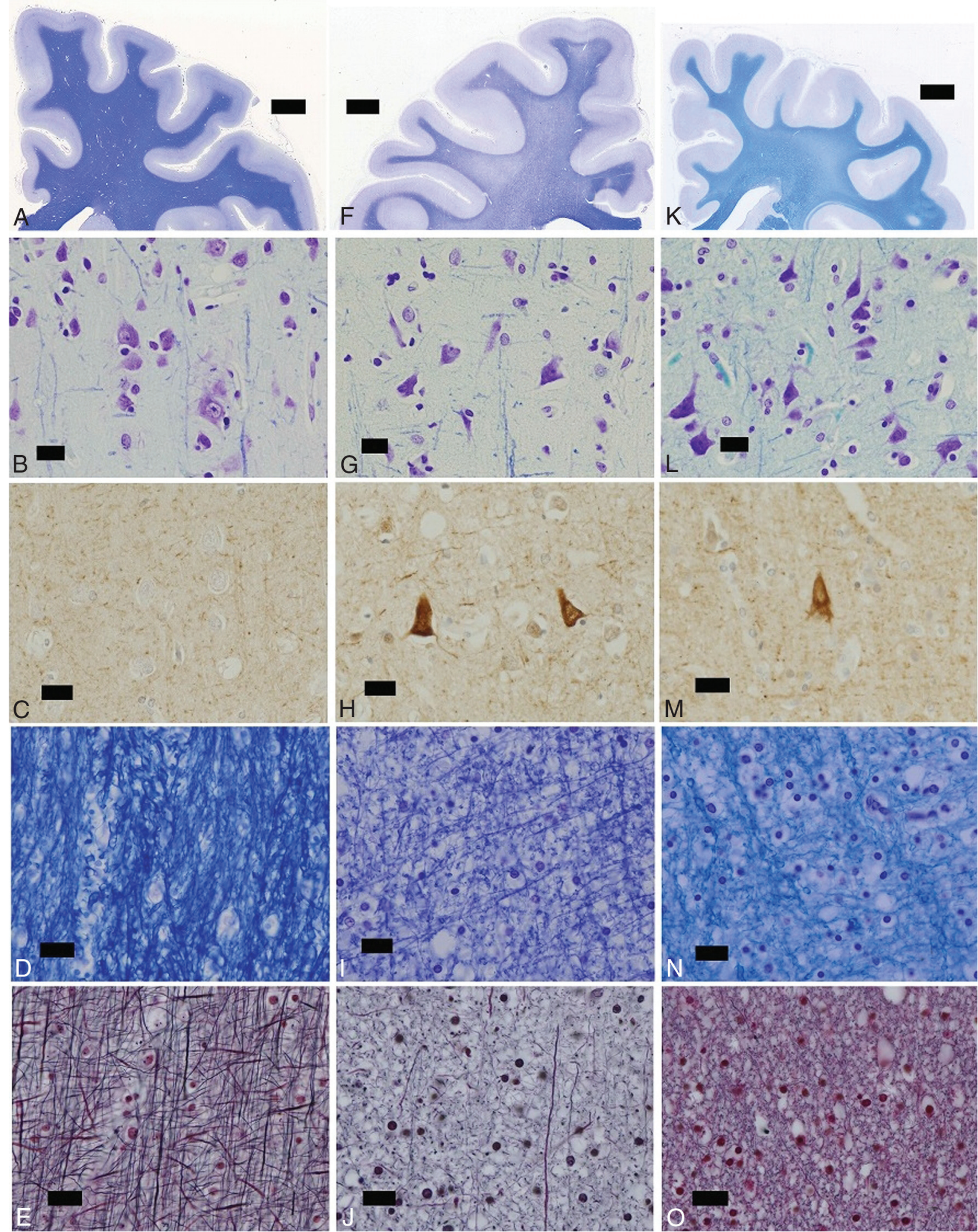

Fig 3. F, Patient 1 shows no evident atrophy of the cerebral cortex and white matter. $G$, The cortical neurons are slightly atrophic. $H$, Accumulation of phosphorylated neurofilaments is noted in several cortical neurons in the fifth layer. I and $J$, The cerebral white matter shows marked loss of myelinated fibers. $K$ and $L$, The cerebral white matter of patient 2 is severely atrophic, and the width of the cerebral cortex is generally reduced. The cortical neurons are shrunken, and the neuropile is markedly atrophic in every layer ( $L$ ). $M$, Some cortical neurons especially in the fifth layer show accumulation of phosphorylated neurofilaments. $N$ and $O$, The cerebral white matter shows moderate loss of myelinated fibers. $A-E$, Control. $F-J$, Patient 1. $K-O$, Patient 2. $A, B, D, F, G, I, K, L, N, K B$ staining; $C, H$, and $M$, phosphorylated neurofilament immunohistochemistry. $E, J$, and $O, B o d i a n$ preparation. 

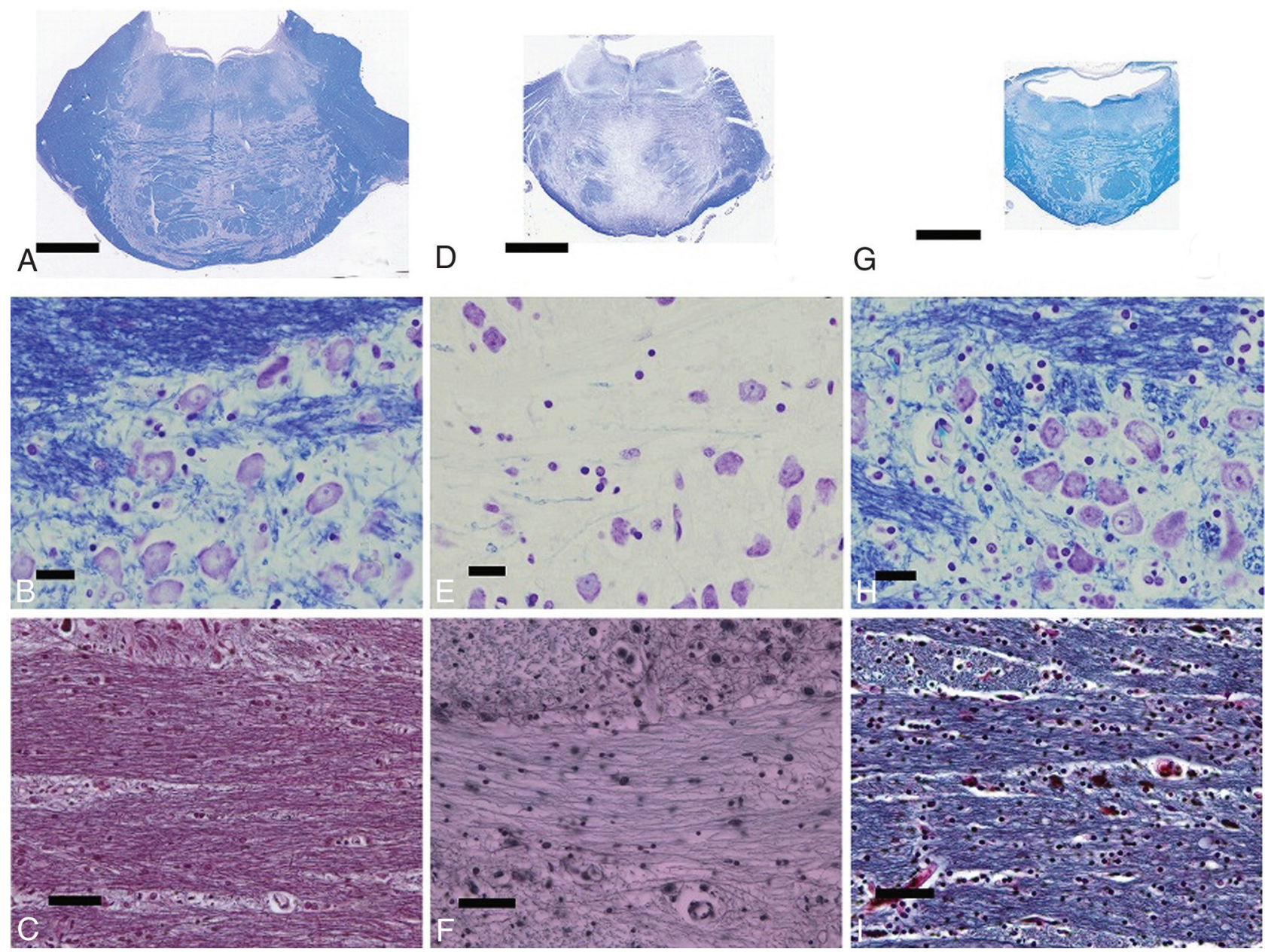

Fig 4. $D-F$, Patient 1 shows moderate atrophy of the pons and marked reduction of myelinated fibers, particularly in the base $(D-F)$ compared with the control $(A-C)$. $G-I$, The pons of patient 2 is markedly atrophic $(G)$, the volume of the neuropile is severely reduced $(H)$, and the attenuation of myelinated fibers in the base is preserved ( $)$. $A-C$, Control. $D-F$, Patient 1. G-I, Patient 2. $A, B, D, E, G, H, K B$ staining. $C, F$, and I, Bodian preparation.

\section{Discussion}

We presented 2 typical cases of patients in a family with DRPLA. The father (patient 1 with adult-onset DRPLA) mainly manifested ataxia, chorea, and dementia with middleaged onset, whereas the son (patient 2 with juvenile-onset DRPLA) mainly manifested ataxia, epilepsy, myoclonus, and severe dementia with young-age onset. MR imaging revealed involvement of the cerebral white matter, which was more prominent in patient 1 , whereas cerebral atrophy was a more prominent feature in patient 2.

The neuropathologic findings in these 2 patients are essentially similar to those reported for other cases of DRPLA, consisting predominantly of combined degeneration of the dentatorubral and pallidoluysian systems. ${ }^{9-12}$ In patient 1 , marked myelinated fiber loss was observed not only in the cerebral white matter but also in the pontine base and tegmentum of the midbrain. In contrast to patient 1 , the myelin loss in patient 2 was slight, though marked atrophy of the cerebral white matter and brain stem were seen. Accumulation of phosphorylated neurofilaments in cortical neurons may indicate the presence of some degenerative process in the neurons in both patients. Given these findings, in patients with DRPLA, high- signal-intensity lesions in the brain stem or the cerebral white matter on T2-weighted images are considered to reflect mainly the loss of myelinated fibers. On the other hand, marked neuronal and neuropile shrinkage in the cerebral cortex was observed in patient 2. Atrophy of the neuropile observed in the cerebral cortex of the patient was considered to be induced by the retardation of the neuronal perikarya, dendrites, dendritic spines, and the presynaptic areas as observed in the mouse model of DRPLA. ${ }^{13}$ These pathologic findings may correlate to the severe cognitive deterioration and epilepsy, which are often observed in patients with juvenile-onset DRPLA.

The MR imaging findings of the cerebral white matter in adult-onset DRPLA resemble those in Binswanger disease. However, we believe that the myelinated fiber loss is not caused by an ischemic mechanism but is a characteristic pathologic finding of DRPLA, because no atherosclerotic changes were found in our present patients. This study revealed that atrophy of the cerebral white matter and pontine base was moderate, but severe loss of myelinated fibers was noted in these regions in adult-onset DRPLA. In contrast, atrophy of the cerebral white matter and pontine base was marked, but the attenuation of myelinated fibers was pre- 
served in these regions in juvenile-onset DRPLA. MR imaging findings seemed to be associated with myelinated fiber loss in DRPLA.

Furthermore, MR imaging findings of the pontine base in adult-onset DRPLA resemble those in central pontine myelinolysis. ${ }^{14}$ However, we considered that the pontine lesions are also caused by the DRPLA itself for the following reasons: 1) No electrolyte disturbance had occurred in our patients, 2) no infiltration of macrophages was observed, 3) slight myelinated fiber loss in the pons was observed also in the patient with juvenile-onset DRPLA, and 4) similar pontine lesions have been observed in many other patients with adult-onset DRPLA. ${ }^{4,14}$

It remains unclarified why myelinated fiber loss appears more severe in adult-onset DRPLA (short expanded CAG repeats) than in juvenile-onset DRPLA (large expanded $C A G$ repeats). Because cerebral white matter involvement in juvenile-onset DRPLA can be observed by MR imaging in the advanced stage, glial pathologic changes may appear more gradually than neuronal degeneration.

\section{References}

1. Naito H, Oyanagi S. Familial myoclonus epilepsy and choreoathetosis: hereditary dentatorubral-pallidoluysian atrophy. Neurology 1982;32:798-807

2. Koide R, Ikeuchi T, Onodera O, et al. Unstable expansion of CAG repeat in hereditary dentatorubral-pallidoluysian atrophy (DRPLA). Nat Genet 1994;6: 9-13
3. Ikeuchi T, Koide R, Tanaka H, et al. Dentatorubral-pallidoluysian atrophy: clinical features are closely related to unstable expansions of trinucleotide (CAG) repeat. Ann Neurol 1995;37:769-75

4. Koide R, Onodera O, Ikeuchi T, et al. Atrophy of the cerebellum and brainstem in dentatorubral-pallidoluysian atrophy: influence of CAG repeat size on MRI findings. Neurology 1997;49:1605-12

5. Miyazaki M, Kato T, Hashimoto T, et al. MR of childhood-onset dentatorubral-pallidoluysian atrophy. AJNR Am J Neuroradiol 1995;16:1834-36

6. Uyama E, Kondo I, Uchino M, et al. Dentatorubral-pallidoluysian atrophy (DRPLA): clinical, genetic, and neuroradiologic studies in a family. J Neurol Sci 1995; 130:146-53

7. Kin T, Hirano M, Taoka T, et al. Global and region-specific analyses of apparent diffusion coefficient in dentatorubral-pallidoluysian atrophy. AJNR Am J Neuroradiol 2006;27:1463-66

8. Kin T, Hirano M, Taoka T, et al. Proton MR spectroscopy of adult-onset dentatorubral-pallidoluysian atrophy. Magn Reson Med Sci 2005;4:123-27

9. Takahashi H, Ohama E, Naito H, et al. Hereditary dentatorubral-pallidoluysian atrophy: clinical and pathologic variants in a family. Neurology 1988;38:1065-70

10. Burke JR, Wingfield MS, Lewis KE, et al. The Haw River syndrome: dentatorubral-pallidoluysian atrophy (DRPLA) in an African-American family. Nat Genet 1994;7:521-24

11. Becher MW, Rubinsztein DC, Leggo J, et al. Dentatorubral and pallidoluysian atrophy (DRPLA): clinical and neuropathological findings in genetically confirmed North American and European pedigrees. Mov Disord 1997;12:519-30

12. Munoz E, Campdelacreu J, Ferrer I, et al. Severe cerebral white matter involvement in a case of dentatorubropallidoluysian atrophy studied at autopsy. Arch Neurol 2004;61:946-49

13. Sakai K, Yamada M, Sato T, et al. Neuronal atrophy and synaptic alteration in a mouse model of dentatorubral-pallidoluysian atrophy. Brain 2006;129: 2353-62

14. Kobayashi J, Nagao M, Kawata A, et al. A case of late adult-onset dentatorubralpallidoluysian atrophy mimicking central pontine myelinolysis. J Neurol 2009;256:1369-71 